\title{
Zur Interpretation des Paramagnetismus der Eisengruppe.
}

Von Otto Laporte in Ann Arbor, Michigan.

Mit 1 Abbildung. (Eingegangen am 10. Februar 1928.)

Die von A. Sommerfeld und dem Verfasser früher gegebenen Formeln für die Suszeptibilität enthalten die Aufspaltungen der Grundterme der Spektren der betreffenden Ionen. In dieser Arbeit werden die Aufspaltungen mittels der relativistischen Dublettformel berechnet. Die auf diesem Wege rein spektroskopisch erhaltenen Magnetonenzahlen stimmen in keinem Falle mit den beobachteten überein. Auch bei den seltenen Erden scheint sich derselbe Effekt bemerkbar zu machen.

Verschiedene mögliche Erklärungen dieser Diskrepanz werden diskutiert.

Vor ungefähr einem Jahre zeigten A. Sommerfeld und der Verfasser*, daß die strenge Gültigkeit des Curieschen Gesetzes $\chi=C / T$ wesentlich von der Gruppierung der Niveaus der einzelnen paramagnetischen Moleküle oder Ionen abhängt. In mehreren wichtigen Arbeiten zeigte darauf Van Vleck**, daß man vom Standpunkt der Wellenmechanik sowohl das Langevinsche Gesetz für den Paramagnetismus als auch die analoge $D$ ebyesche Formel für die Molekularrefraktion ableiten kann. Der Van Vlecksche Beweis, der in weitem Maße vom Modell unabhängig ist, hat nur zur Voraussetzung, daß in den in Frage kommenden, mit permanenten magnetischen oder elektrischen Momenten begabten individuellen Systemen keine dem Grundterm so nahe benachbarten angeregten Zustände vorkommen, die zu Frequenzen Anlaß geben, welche von der Größenordnung $k T / h$ sind.

Da, wie in der oben erwähnten Arbeit gezeigt wurde, solche natürliche mit $k T / h$ vergleichbare Frequenzen in der Tat in der Eisengruppe vorkommen, ist zur Interpretation der dort gemessenen Magnetonenzahlen nicht nur die Kenntnis der tieferen Terme der betreffenden Ionen notwendig, sondern auch die genaue Kenntnis der Energiedifferenzen zwischen den verschiedenen Termen.

* 0. Laporte und A. Sommerfeld, ZS. f. Phys. 40, 333, 1926.

** J. H. Van Vleck, Phys. Rev. 29, 727, 1927. 
Es sei hier gleich bemerkt, daß dieser die Interpretation der Magnetonenkurve der Eisengruppe so sehr erschwerende Umstand für die seltenen Erden nicht (oder nur unwesentlich; vgl. S. 768) berücksichtigt zu werden braucht, da die Aufspaltungen wegen der viel höheren Kernladung bedeutend größer als $k T / h$ geworden sind. Die überraschend gute Übereinstimmung der Hund schen* theoretischen Magnetonenkurve mit den gemessenen Werten für die seltenen Erden sehen wir als deutlichen Beweis an, $\mathrm{da} ß$ diese auf die Theorie der Serienspektren aufbauende Interpretation im Prinzip richtig ist. Es ist wichtig, festzustellen, daß sich in der Tat die Quantenzahlen der tieferen Terme der Ionen auch in Lösung erhalten und daß der Einfluß der Anionen sowie der Moleküle des Lösungsmittels nur geringfügig ist. Wir wünschen dies besonders im Hinblick auf Versuche von Joos* und von Bose*** hervorzuheben, welche die Interpretation der Magnetonenzahlen auf ganz anderer Basis versuchen.

Die Ableitung der nötigen Formeln sei in Kürze gegeben. Wir gehen aus von der Energie eines Atoms oder Ions im Magnetfeld:

$$
\varepsilon_{j, m}=m g(j, l, s) \mu_{B} H,
$$

wo $m$ die magnetische Quantenzahl, $g$ den Landéschen Aufspaltungsfaktor, $\mu_{B}$ das Bohrsche Magneton und $H$ die Feldstärke bedeutet. Die Energiedifferenz zwischen den einzelnen durch $j$ unterschiedenen Niveaus eines Termmultipletts sei

$$
\varepsilon_{j}=h c \Delta \nu_{j}
$$

wo $\Delta v_{j}$ den Abstand vom tiefsten Niveau des Terms bedeutet. Dann wird die mittlere magnetische Energie, d. h. das Produkt von Magnetisierung $M$ und Feldstärke $H$ von $N$ Ionen:

$$
\frac{M H}{N}=\frac{\chi H^{2}}{N}=\frac{\sum j \sum \varepsilon_{j, m} e^{-\frac{\varepsilon_{j, m}+\varepsilon_{j}}{k T}}}{\sum j \sum_{m} e^{\frac{\varepsilon_{j, m}+\varepsilon_{j}}{k T}}} .
$$

* F. Hund, ZS. f. Phys. 33, 855, 19:5.

** G. Joos, Ann. d. Phys. 81, 1076, 1926.

*** D. M. Bose, ZS. f. Phys. 43, 864, 1927. Dieser Verfasser sieht als Hauptvorteil seiner Theorie die Erklärung des von der Art des Metalls unabhängigen Einstein-de Haas-Effekts an. Dieser scheint jedoch eher dadurch zustande zu kommen, daß das Verhältnis der magnetischen und mechazischen liomente der freien Leitungselektronen, nicht der Tetallatome gemessen wird. 
Durch Entwickeln der e-Funktion nach Potenzen von $H$ erhält man in erster Näherung:

$$
\chi=\frac{\mu_{\text {eff. }}^{2}}{3 k T} ; \quad \mu_{\text {eff. }}^{2}=\mu_{B}^{2} \frac{\sum j j(j+1) g_{j}^{2} \cdot(2 j+1) e^{-\frac{h c \Delta v_{j}}{k T}}}{\sum j(2 j+1) e^{-\frac{h c \Delta v_{j}}{k T}}},
$$

was mit der l. c. gegebenen Formel übereinstimmt*.

Wegen des Auftretens der $\boldsymbol{\Delta} \boldsymbol{v}_{j}$ wäre im Prinzip also zur Berechnung der Magnetonenzahlen in der Eisengruppe eine genaue Kenntnis der Spektren der zwei- und dreifach ionisierten Atome nötig. Die Hundsche Theorie der Spektren gibt in der Tat eine genaue Voraussage über den Charakter des tiefsten Terms, d. h. über die Quantenzahlen $l, s, j$. Die Berechnung der A ufspaltungen $\boldsymbol{\Delta} v_{j}$ wurde dagegen (l.c.) durch Durchrechnung der zwei Grenzfälle $h c \Delta v_{j} \gg k T$ und $h c \Delta \nu_{j} \ll k T$ umgangen. Auf diese Weise ergaben sich zwei Magnetonenkurven für sehr hohe und sehr niedrige Temperaturen. Bis auf zwei lagen in der Tat alle beobachteten Magnetonenwerte zwischen diesen beiden Kurven.

Die in letzter Zeit erzielten großen Fortschritte in der Entwirrung höherer Funkenspektren haben nun gezeigt, daß die Sommerfeldsche relativistische Dublettformel

$$
\frac{\Delta v}{R}=\frac{(Z-\sigma)^{4}}{n^{3} l(l+1)}
$$

die Aufspaltungen homologer Terme in Funkenspektren gleicher Elektronenzahl gut wiederzugeben vermag. Dadurch wird es jetzt möglich, die in (4) vorkommenden $\Delta \boldsymbol{v}_{j}$ für die höheren Funkenspektren aus denen der weniger ionisierten Spektren zu berechnen und einen genauen Vergleich der Theorie mit der Erfahrung auszuführen.

* Es sei hier auf folgenden Fall aufmerksam gemacht. Nehmen wir die Separation $\Delta \nu_{j}$ als sehr klein an, selhst verglichen mit der durch das Feld hervorgerufenen Aufspaltung, dann muß man in obigen Formeln $\varepsilon_{m}$ durch

$$
{ }^{e_{m_{s}}, m_{l}}=\left(2 m_{s}+m_{l}\right) \mu_{B} H
$$

and die einfachen Summen überall durch doppelte nach $m_{s}$ and $m_{l}$ ersetzen. Man erhält dann

$$
\mu_{\text {eff. }}^{2}=\mu_{B}^{2}[4 s(s+1)+l(l+1)] \text {. }
$$

Tatsächlich sind aber die in der Eisengruppe vorkommenden $\Delta \nu_{j}$, obwohl sie mit $k T / h c$ vergleichbar sind, immer grof gegen $\mu H$. Um bei der uns interessierenden Grölenordnung der $\Delta \nu$ die obige Formel anwenden zu dürfen, müßte man außerdem zu sehr hohen Temperaturen gehen, um das Entwickeln der e-Funktion zu rechtfertigen. Obige von $\mathrm{VanVleck}$ gegebene Forme] scheint auf unseren Fall nicht anwendbar. 
Obwohl es für Spektren mit nur wenigen Elektronen, d.h. am Anfang der Periode, zweckmäßiger erscheint, statt der Sommer feld schen Dublettformel (5) die Landésche, welche $(Z-\sigma)^{4}$ durch $Z_{i}^{2} Z_{a}^{2}$ ersetzt, zu benutzen, haben wir uns im folgenden durchweg der ersteren bedient, selbst wenn man dadurch einen stärkeren Gang der Abschirmungszahlen in Kauf nehmen mub. Es geschah das, um alle $\sigma$-Werte von der gleichen Gröhenordnung zu haben.

Die Terme, die die Hundsche Theorie für die Normalzustände der einzelnen Ionen voraussagt, sind bzw. für $0,1,2,3 \ldots d$-Elektronen:

$$
{ }^{1} \mathrm{~S},{ }^{2} \mathrm{D},{ }^{3} \mathrm{~F},{ }^{4} \mathrm{~F},{ }^{5} \mathrm{D},{ }^{6} \mathrm{~S},{ }^{5} \mathrm{D},{ }^{4} \mathrm{~F},{ }^{3} \mathrm{~F},{ }^{2} \mathrm{D},{ }^{1} \mathrm{~S} .
$$

Sie sind in der ersten Hälfte der Periode, d. h. bis zum ${ }^{6} S$-Term, regelrecht, in der zweiten verkehrt.

Nach einer noch unpublizierten Theorie von Goudsmit müssen die Aufspaltungen der für zwei, drei, sieben und acht Elektronen als Grundterme erscheinenden F-Terme mit $\frac{5}{7}$ multipliziert, d. h. gleichsam vom $l$-Wert 3 auf den $l$-Wert 2 reduziert werden, um eine Anwendung von (5) zu ermöglichen.

Tabelle 1.

\begin{tabular}{|c|c|c|c|c|c|}
\hline $\begin{array}{c}\text { Konfiguration } \\
d^{z}\end{array}$ & Term & Spektrum & $\Sigma \Delta v_{j}$ & $\sigma$ & ${ }^{\mu} W$ \\
\hline$d^{\mathfrak{l}}$ & ${ }^{2} D$ & $\begin{array}{l}\mathrm{K} \\
\mathrm{Ca}^{+} \\
\mathrm{Se}^{++} \\
\mathrm{Ti}^{+++}\end{array}$ & $\begin{array}{c}2,74 \\
60,8 \\
197 \\
384,5\end{array}$ & $\begin{array}{l}16.08 \\
13,60 \\
12,40 \\
11,83\end{array}$ & $\begin{array}{c}- \\
\overline{12,4} \\
10,4\end{array}$ \\
\hline$d^{2}$ & ${ }^{3} F$ & $\begin{array}{l}\mathrm{Sc}^{+} \\
\mathrm{Ti}^{++}\end{array}$ & $\begin{array}{l}184,9 \\
421,9\end{array}$ & $\begin{array}{l}13,21 \\
12,44\end{array}$ & $\overline{15,8}$ \\
\hline$d^{3}$ & ${ }^{4} F$ & $\begin{array}{l}\mathrm{Ti}^{+} \\
\mathrm{V}^{++} \\
\mathrm{Cr}^{+++} \\
\mathrm{Mn}^{++++}\end{array}$ & $\begin{array}{c}307,6 \\
580 \\
912 \\
1270\end{array}$ & $\begin{array}{l}13,17 \\
12,64 \\
12,45 \\
12,20\end{array}$ & $\begin{array}{l}\overline{16,1} \\
13,0 \\
10,0\end{array}$ \\
\hline$d^{4}$ & ${ }^{5} D$ & $\begin{array}{l}\nabla^{+} \\
\mathrm{Cr}^{++} \\
\mathrm{Mn}^{+++}\end{array}$ & $\begin{array}{l}339,3 \\
566 \\
852\end{array}$ & $\begin{array}{l}13,17 \\
12,8 \\
12,6\end{array}$ & $\begin{array}{l}-\overline{18,6} \\
15,7\end{array}$ \\
\hline$d^{6}$ & ${ }^{5} D$ & $\begin{array}{l}\mathrm{Mn}^{+} \\
\mathrm{Fe}^{++}\end{array}$ & $\begin{array}{l}635,3 \\
998\end{array}$ & $\begin{array}{l}13,48 \\
13,1\end{array}$ & $\overline{32,3}$ \\
\hline$d^{7}$ & ${ }^{4} F$ & $\begin{array}{l}\mathrm{Fe}^{+} \\
\mathrm{Co}^{++}\end{array}$ & $\begin{array}{l}1244,9 \\
1890\end{array}$ & $\begin{array}{l}13,24 \\
13,1\end{array}$ & $\overline{32,8}$ \\
\hline$d^{8}$ & ${ }^{3} F$ & $\begin{array}{l}\mathrm{Co}^{+} \\
\mathrm{Ni}^{++}\end{array}$ & $\begin{array}{l}1650 \\
2347\end{array}$ & $\begin{array}{l}13,55 \\
13,31\end{array}$ & $\overline{27,8}$ \\
\hline$d^{9}$ & ${ }^{2} D$ & $\begin{array}{l}\mathrm{Ni}^{+} \\
\mathrm{Ca}^{++}\end{array}$ & $\begin{array}{l}1530 \\
2130\end{array}$ & $\begin{array}{l}13,65 \\
13,4\end{array}$ & $\overline{17,64}$ \\
\hline
\end{tabular}


Die so erhaltenen Abschirmungskonstanten $\sigma$, Aufspaltungen $\Delta \nu$ und [nach (4) berechneten] Weissschen Magnetonenzahlen $\mu_{W}$ sind in der vorstehenden Tabelle 1 zusammengestellt. Extrapolierte Werte sind durchweg kursiv gedruckt. Auf folgendem Wege wurde die Rechnung ausgeführt: In den meisten Fällen ist der zur Konfiguration $d^{z}$ gehörige Term im ersten Funkenspektrum beobachtet. Die Summe seiner Aufspaltungen findet sich in der vierten Spalte. Sodann wurden mittels Formel (5) - nach eventueller Multiplikation mit $\frac{5}{7}$ - die zugehörigen Abschirmungszahlen б berechnet und auf höhere Funkenspektren extrapoliert. Bei dieser Extrapolation wurde nicht nur die bekannte langsame

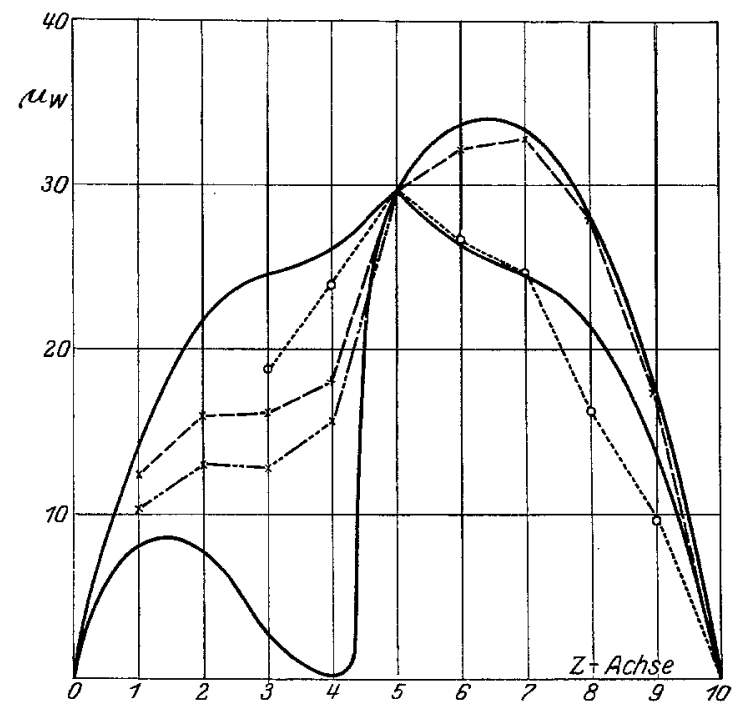

Fig. 1.

Abnahme der $\sigma$ korrespondierender Terme mit zunehmender Kernladung in Betracht gezogen, sondern auch die Änderung der $\sigma$ in entsprechenden Termreihen vom Typus $\left(d^{z-1} s\right)$ und $\left(d^{z-2} s^{2}\right)$, deren Aufspaltungen in zahlreichen Funkenspektren bekannt sind*. Nur für acht bzw. neun Elektronen sind die betreffenden Terme ${ }^{3} F$ bzw. ${ }^{2} D$ überhaupt noch in keinem Spektrum aufgefunden worden. Unsere in diesen Fällen vielleicht etwas unsichere Extrapolation hat aber auf die in der letzten Spalte angegebenen $\mu_{W}$-Werte nur geringen Einfluß. Wie man aus der gleich zu beschreibenden Fig. 1 ersieht, sind für acht und neun $d$-Elektronen die berechneten $\mu_{W}$-Werte gegen eine Änderung der $\Delta \nu$ nur wenig empfindlich.

* Vgl. mehrere Arbeiten von R. C. Gibbs und H. E. White, Phys. Rev. 1927. 
Unsere jetzige Figur ist der 1. c. mitgeteilten ähnlich. Die beiden ausgezogenen Kurven stellen wie früher die theoretischen Werte $\mu_{W}$ für $h c \Delta v \gg h T$ und $h c \Delta v \ll k T$ dar. Die durch lang gestrichelte bzw. strichpunktierte Linien verbundenen Kreuzchen sind die in der sechsten Spalte der Tabelle angegebenen berechneten $\mu_{W}$-Werte. Die experimentellen Werte, dieselben wie in der früheren Arbeit, sind durch Kreise dargestellt, die durch fein gestrichelte Linien verbunden sind.

Wie man sieht, kann von Übereinstimmung in keinem der Fälle, wo ein vielfacher Term vorliegt, die Rede sein. Nur für die Konfiguration $d^{5}$, die einen ${ }^{6} S$-Term, einen Einfachterm, zur Folge hat, ergibt sich, wie auch schon l. c. hervorgehoben wurde, Übereinstimmung. Man kann die allgemeine Folgerung ziehen, dab, obwohl der Einfluß der höheren Niveaus eines Multipletterms auf den $\mu_{W}$-Wert ein wesentlicher sein muB, die Mittelung nach (4) doch in keinem Falle auch nur zu annähernder Übereinstimmung führt. Die berechneten Werte liegen in der ersten Hälfte der Periode zu tief, in der zweiten zu hoch, verglichen mit den gemessenen. Man kann die Abweichung auch so formulieren, daß die nach (4) berechnete Anzahl Ionen im $j$-ten Teilniveau

$$
n_{j}=(2 j+1) e^{-\frac{\varepsilon_{j}}{k T}}
$$

zu klein ist; in Wirklichkeit befinden sich in den höheren Niveaus mehr Ionen als nach obiger Formel.

Man kann diesen Tatbestand auf verschiedene mehr oder weniger unbefriedigende Weisen zu erklären versuchen. Es könnte behauptet werden, daß, obwohl die nach (5) berechneten Aufspaltungen im gasförmigen Zustand richtig sind, im flüssigen oder festen Zustand die verschiedenen Niveaus nicht so genau, jedenfalls nicht was ihren Abstand betrifft, erhalten $z u$ sein brauchen. Unbefriedigend ist an diesem Erklärungsversuch, daß man nicht einsieht, warum die ${ }^{5} D$ - und ${ }^{4} F$-Terme, die für sechs und sieben Elektronen als Grundterme erscheinen, im flüssigen oder festen Zustand so gut wie gar nicht aufgespalten sein sollen, und warum für acht und neun Elektronen in den Termen sogar die Reihenfolge der Niveaus umgekehrt wie im Gaszustand sein soll. Andererseits muß aber doch eine gewisse Veränderung der Terme und Niveaus beim Übergang aus dem gasförmigen in den flüssigen Aggregatzustand stattfinden. Denn nur so kann man sich die zuerst von Joos hervorgehobene Tatsache erklären, daß, obwohl die Albsorption der Lösungen der Salze der Eisengruppe in Sichtbaren liegt, sicherlich keines der in Frage kommenden Funkenspektren Absorptionslinien mit einer Wellen- 
länge größer als $1700 \AA$ A.-E. aufweist. Überhaupt ist es nur plausibel, daß die (um mehrere Volt) höheren Terme im Spektrum eines Ions in Lösung beträchtlich verändert, z. B. dem Grundzustand näher gerückt werden, da solche Terme doch zu viel weniger stabilen, leichter beeinflußbaren Zuständen gehören. So erklärt sich auch der von Joos (l. c.) erwähnte Zeemaneffekt der Absorptionslinien der Lösungen seltener Erden, der durchaus von dem im Gaszustand zu erwartenden abweicht. Wie indessen schon hervorgehoben wurde, verbieten die erfolgreiche Berechnung der Magnetonenzahlen der seltenen Erden und die vielfachen Bestätigungen der zu dieser Berechnung benutzten Pauli-Hundschen Theorie die Annahme der von Joos vertretenen Anschauung, daß in Salzlösungen überhaupt nur der Magnetismus und die Absorption von Komplex-Ionen, nicht der Metallkationen gemessen werden.

Des weiteren könnte man Einwände gegen den Gebrauch der Verteilungsformel (6) wegen der Metastabilität der höheren Niveaus eines Terms richten. Man sollte meinen, daß wegen des metastabilen Charakters tatsächlich eine Anhäufung von Ionen in diesen Zuständen stattfinden würde, da die einmal in diesen Zustand gekommenen Ionen keine Gelegenheit haben, in den Grundzustand zurückzukehren. Indessen überlegt man sich leicht, daß ein solches Verhalten ebensosehr die Intensitätsregeln der Multipletts verändern müßte, besonders die OrnsteinDorgelosche Summenregel. Doch sind gerade diese Regeln durch zahlreiche Messungen sichergestellt; kleine Abweichungen von einer für uns gar nicht zu beachtenden Größenordnung können ungezwungen durch beginnende Abweichung von der Russell-Saundersschen Kopplung zu anderen Kopplungsschemas erklärt werden.

Man könnte schlielilich noch einen prinzipiellen Einwand gegen die Art der benutzten Statistik und damit gegen (6) richten. Die für Ionen zweifellos zu benutzende Fermi-Dirac-Statistik verknüpft, wie $\mathrm{Pauli}$ * gezeigt hat, aufs engste die Verteilung über die verschiedenen Translationsenergieniveaus $\varepsilon_{s}$ und über die Energieniveaus des individuellen Teilchens, $\varepsilon_{j}$ und $\varepsilon_{m}$. Es dürfen nämlich nur solche Ionen gleiche $j$ - und $m$-Werte haben, die verschiedene Translationsenergien besitzen. Es ist nun durchaus denkbar, da 6 im flüssigen und besonders im festen Zustand die Tranlationsbewegungsmöglichkeiten so eingeschränkt sind, daß die Verteilung über die Niveaus des individuellen Ions nicht mehr der Maxwell-Boltzmannschen entspricht, sondern daß mehr Ionen in den

* W. Pauli, ZS. f. Phys. 41, 81, 1927.

Zeitschrift für Physik. Bd. 47. 
höheren Zuständen sind. Dies würde dem Verhalten eines der FermiDiracschen Statistik folgenden Gases bei sehr tiefen Temperaturen entsprechen, wo ja auch mehr Molekïle in höheren Niveaus $\varepsilon_{8}$ sich befinden, als einer reinen Boltzmannverteilung entspräche (Nullpunktsenergie und -druck). Andererseits spricht gegen diese Auffassung die weitgehende Unabhängigkeit der paramagnetischen Suszeptibilität, sowohl vom chemischen Charakter des Anions (z. B. Chlorid oder Sulfat) als anch vom Aggregatzustand (festes Salz oder verdünnte Lösung).

Eine Diskrepanz im gleichen Sinne, wie sie in der Eisengruppe vorliegt, findet sich übrigens auch in der Magnetonenkurve der seltenen Erden. Wie schon erwähnt, konnte Hund gute Übereinstimmung mit den experimentellen Werten erzielen unter der Annahme, daß die Aufspaltungen der Grundterme groh sind verglichen mit $k T / h$. Nur im Falle des $\mathrm{Eu}^{+++}$-Ions ergab sich eine größere Abweichung, die 1. c. ebenfalls durch Hereinspielen der höheren Niveaus zu deuten versucht wurde. Es ist aber nicht schwierig, diesen Einfluß quantitativ zu schätzen, obwohl noch keine Spektren der seltenen Erden entwirrt sind. Denn ebenso wie die in Tabelle 1 auf $\mathrm{S} .764$ angegebenen Abschirmungszahlen alle in der Nähe der Abschirmungszahl des Röntgendubletts $M_{39} M_{3 \mathrm{a}}$ liegen, mul es auch möglich sein, in erster Näherung die Aufspaltungen des in $\mathrm{Eu}^{+++}$den Normalzustand repräsentierenden ${ }^{7} F\left(f^{6}\right)$-Terms aus der Abschirmungszahl des Dubletts $N_{43} N_{44}$ zu erhalten. Indem wir für diese letztere den üblichen Wert 34 einsetzen, erhalten wir für die Totalaufspaltung $\Delta^{7} F$ etwa 5400 und nach (4) für die Magnetonenzahl in Weisseinheiten :

$$
\boldsymbol{\mu}_{W}=8,51
$$

während als beobachtete Werte

$$
15,5 \text { und } 17,9
$$

angegeben werden. Der berechnete Wert ist viel zu klein. Die Abweichung ist also in derselben Richtung und von derselben Größenordnung wie in der Eisengruppe. Es scheint sich also hier um einen allgemeinen Effekt zu handeln, der sowohl in der Eisengruppe wie in den seltenen Erden auftritt.

In diesem Zusammenhang sei noch auf eine ähnliche Diskrepanz hingewiesen, die von $K u h n *$ in seinen Untersuchungen über die anomale Dispersion von Metalldämpfen entdeckt wurde. Aus der Magnetorotation konnte $\mathrm{Kuhn}$ direkt die Anzahl Atome in einem bestimmten Quanten-

* W. Kuhn, Danske Vid. Selskab 7, Nr. 12, 1926. 
zustand bestimmen. Er fand nun für Thalliumdampf einerseits die Zahl der bei einer bestimmten Temperatur in den beiden tiefen Termen ${ }^{2} \boldsymbol{P}_{1_{\mathbf{2}}}$ und ${ }^{2} P_{3 / 2}$ sich befindenden Atome, andererseits berechnete er das Verhältnis der Besetzungszahlen der beiden Niveaus aus dem Boltzmannschen Verteilungsgesetz. Die beiden so ermittelten Werte für $n_{3 / 2} / n_{1 / 2}$ stimmten nicht miteinander überein; und zwar ergab sich nach der Quantenstatistik ein etwa fünfmal kleinerer Wert. In Wirklichkeit schienen also fünfmal mehr Atome sich im höheren Zustand ${ }^{2} P_{3 / 2}$ zu befinden, als nach dem Verteilungsgesetz vorausgesehen war. Eine Deutung der Erscheinung wurde von Kuhn nicht gegeben. Diese von Kuhn bemerkte Abweichung von der Boltzmannverteilung liegt in derselben Richtung wie die von uns diskntierte in der Eisen- und Erdengruppe. Zum Schluß sei noch einmal die Wichtigkeit neuer Messungen hervorgehoben, die die Abhängigkeit der Magnetonenzahlen von der Temperatur, die durch (4) gefordert ist, nachprüfen sollen. Wenn auch, wie wir gesehen haben, der Absolutwert des magnetischen Moments durch die hier gegebenen Formeln nicht richtig wiedergegeben wird, so ist es doch wichtig, zu erfahren, ob in der Tat für höhere oder niedrige Temperaturen die beiden Grenzkurven erreicht werden. Auch die spezifische Wärme der Salze der Eisengruppe sollte den Einfluß der höheren Niveaus des Grundterms des Ions zeigen, wovon man sich durch zweimalige geeignete Differentiation der Zustandssumme $\sum g_{j} e^{-h c \Delta v_{j} / k T}$ nach der Temperatur überzeugt. Durch Messung der spezifischen Wärme müßten sich viele der hier aufgeworfenen Fragen entscheiden lassen, insbesondere. die Frage, wieweit die im Gaszustand als Spektralterme auftretenden Energiezustände auch im festen oder flüssigen Zustand vorhanden sind.

Herrn Stud. R. F. Bacher, der die vielen numerischen Rechnungen ausfährte, sei auch an dieser Stelle herzlichst gedankt. Den Herren Dennison, Goudsmit, Oppenheimer und Uhlenbeck bin ich für viele Ratschläge und Diskussionen zu großem Danke verpflichtet.

Ann Arbor, Mich., U.S.A., University of Michigan Januar 1928. 